\title{
Quantum Stabilization of General-Relativistic Variable-Density Degenerate Stars
}

\author{
David Eric Cox ${ }^{1}$, Ronald L. Mallett ${ }^{1}$, M. P. Silverman ${ }^{2}$ \\ ${ }^{1}$ Department of Physics, University of Connecticut, Storrs, USA \\ ${ }^{2}$ Department of Physics, Trinity College, Hartford, USA \\ Email: mark.silverman@trincoll.edu
}

Received April 23, 2012; revised May 18, 2012; accepted June 3, 2012

\begin{abstract}
Research by one of the authors suggested that the critical mass of constant-density neutron stars will be greater than eight solar masses when the majority of their neutrons group into bosons that form a Bose-Einstein condensate, provided the bosons interact with each other and have scattering lengths on the order of a picometer. That analysis was able to use Newtonian theory for the condensate with scattering lengths on this order, but general relativity provides a more fundamental analysis. In this paper, we determine the equilibrium states of a static, spherically-symmetric variable-density mixture of a degenerate gas of noninteracting neutrons and a Bose-Einstein condensate using general relativity. We use a Klein-Gordan Lagrangian density with a Gross-Pitaevskii term for the condensate and an effective field for the neutrons. We show that a new class of compact stars can exist with masses above the Oppenheimer-Volkoff limit, provided the scattering length of the bosons is large enough. These stars have no internal singularities, obey causality, and demonstrate a quantum mechanism consistent with general relativity that could prevent collapsed stars from becoming black holes.
\end{abstract}

Keywords: Gravity; General Relativity; Neutron Stars; Black Holes; Bose-Einstein Condensates; Numerical Physics

\section{Introduction}

The general theory of relativity (GR) predicts that a sufficiently-massive non-rotating electrically-neutral neutron star modeled as a noninteracting degenerate gas will collapse to a geometric point within an event horizon: a black hole [1]. The critical mass for these Oppenheimer-Volkoff $(\mathrm{OV})$ stars is $0.71 M_{\odot}$. Taking rotation and particle interaction into account raises the critical mass to at best $3 M_{\odot}$ [2]. Surveys of stellar black hole candidates with both low- and high-mass companions suggest maximum masses of $45 M_{\odot}[3,4]$. The prediction that massive stars must collapse to nonphysical end states is a consequence of GR's classical limits. Presumably, once the star has collapsed to the order of the Planck length $\left(1.62 \times 10^{-35} \mathrm{~m}\right)$, quantum effects will dominate, preventing further collapse. Although this assumption avoids a singularity, the resulting prediction of objects with $10^{57}$ particles compressed into a $10^{-105} \mathrm{~m}^{3}$ volume is unpalatable to many.

However, if there is a natural process that consistently prevents massive stars from collapsing, then GR is not inconsistent with physically-sensible expectations. Pressure is a gravitational source in GR, so lowering the star's pressure would raise its critical mass. Adding rot- ation and neutron interaction approximately quadrupled the critical mass of an OV star. Assuming it would have the same effect on the stars we are about to discuss (and confirming this is the subject of future inquiries), then if the modified critical mass is above $12 M_{\odot}$, we would not expect the formation of stellar black holes. While this would not prevent the formation of intermediate and super-massive black holes, it would remove one category of unsettling astronomical objects.

One of the authors proposed a mechanism that lowers stellar pressure [5]. If even-numbered groups of neutrons condense into spin- 0 bosons in a manner analogous to Cooper pairing, and if these bosons formed a BoseEinstein condensate instead of a superfluid, then the condensate would exert less pressure than the remaining neutrons, reducing the star's gravitational field enough to ensure stability. Note that this is a different process than previous models which have shown that pion or kaon condensates increase pressure. The presence of these exotic Bose-Einstein condensates, which reduce the critical mass, have been ruled out by recent measurements of neutron star masses on the order of two solar masses [6]. These measurements do not rule out the possibility of Bose-Einstein condensates consisting of groups of neutrons, provided such condensates increase 
neutron-star critical mass.

The proposed model had $s$ neutrons condense into bosons with masses,

$$
m_{\mathrm{b}}=Q s m,
$$

where $m$ is the neutron mass and $Q$ reflects the strength of the neutron-neutron interaction that produces the bosons. The remaining neutrons sink to the star's center while the condensate forms a halo. The two materials are in hydrostatic and chemical equilibrium. This model used two simplifying assumptions. One is that each material has a constant density and the other is that the gravitational field is weak enough to use the Newtonian equation for hydrostatic equilibrium instead of the TOV equation. The results for bosons that interact with each other with scattering lengths on the order of a picometer justified these assumptions.

For chemical equilibrium,

$$
s \mu_{\mathrm{n}}=\mu_{\mathrm{b}}
$$

must hold true at the boundary between the neutrons and the condensate. The chemical potential of a Bose-Einstein condensate, deduced from low-temperature experiments on Bose gases, takes the form,

$$
\mu_{\mathrm{b}}=m_{\mathrm{b}} c^{2}\left(1+4 \pi \lambda_{\mathrm{b}}^{2} a n_{\mathrm{b}}\right),
$$

with reduced Compton length, $\lambda_{\mathrm{b}} \equiv \hbar /\left(m_{\mathrm{b}} c\right)$, scattering length, $a$, and number density, $n_{\mathrm{b}}$ [7-10].

Figure 1 shows some of the end-states, called fermicon (fermions and a boson condensate) stars. In the $Q s=2.2$, $a=-100$ am case, there is a critical mass of $3.19 M_{\odot}$ compared to the OV critical mass of $0.71 M_{\odot}$. The critical masses for fermicon stars with scattering lengths of \pm 1 pm is greater than $8 M_{\odot}$, although it is difficult to say how much greater from the obtainable solutions.

Unfortunately, the $a=-100$ am case is suspect because the TOV equation would be more appropriate since the radii of the stars are comparably close to the event horizons they would have had if they collapsed to black holes (stars with the critical mass have radii only $6 \%$ greater than their Schwarzschild radii). In this article

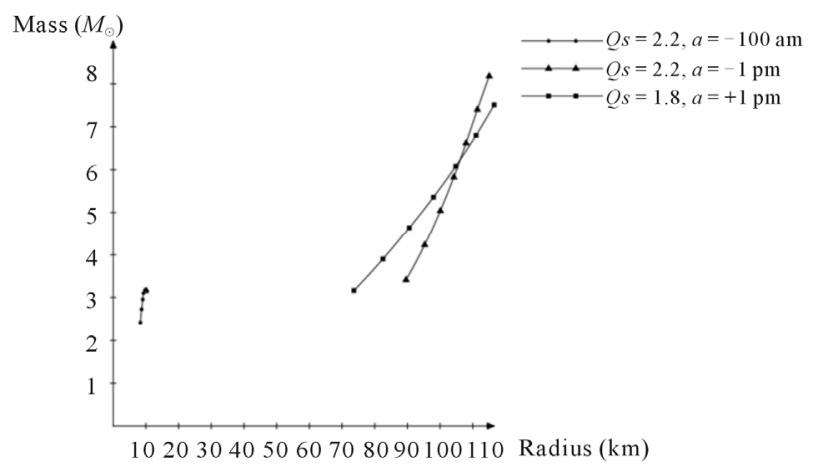

Figure 1. Mass versus radius in type I fermicon stars. we remove the restrictions of the previous investigation and study variable-density stars using GR.

The fermicon model should not be confused with analyses that have examined effects of an external scalar field on the critical mass of a neutron star. Such research is motivated, for example, by the possibility that scalar fields can represent dark matter or other exotic quantities and explore the consequences of their presence on stellar stability. Although scalar fields are present in our models, they represent quantities intrinsic to the star, which is assumed to occupy empty space. Furthermore, these fields are the result of neutron grouping to a BoseEinstein condensate. The grouping of neutrons into superfluid states is believed to occur within neutron stars [6], and the possibility that superfluid states can change to Bose-Einstein condensates has been established in terrestrial laboratories [11]. As a result, the experimental basis of our models is more solidly established.

Our general-relativistic model qualitatively differs from the original one in two aspects. First, the original model, which gave solutions for what we term type I fermicon stars, assumed that the condensate and remaining neutrons physically separate with the neutrons occupying the core (an "oil-and-water" model), while our general-relativistic model, which gives solutions for what we call type II fermicon stars, assumes that the condensate and remaining neutrons are mixed together (a "salt-water" model). Solutions existed for type I fermicon stars because they are two-body problems in Newtonian theory. In GR, the gravitational field counts as a "body" since it can feel its own effects and the type I fermicon model becomes a three-body problem. The "salt-water" model of type II fermicon stars simplifies the problem back to a two-body (star plus field) one.

Second, type I fermicon stars included those whose bosons attracted each other. Our initial investigations of self-interacting boson stars using GR suggest that mutually-attractive bosons reduce the critical mass, so type II fermicon stars only have bosons with repulsive self-interaction $(a>0)$ formed from exothermic grouping $(Q<1)$.

Neutrons with the same momentum eigenstates can pair. These pairs do not need to be adjacent to each other in physical space. The Pauli exclusion principle does not allow more than two neutrons (one spin up and one spin down) to have the same momentum eigenstates, so the majority of neutrons in a system have high-momentum eigenvalues and correspondingly high energies.

If the pairs behave like spin-0 bosons, then these bosons can all occupy a ground-state energy, $E$, with a corresponding ground-state momentum eigenvalue,

$$
p_{\text {ground }}=\sqrt{(E / c)^{2}-\left(m_{\mathrm{b}} c\right)^{2}} .
$$


All of the neutrons which have momentum eigenvalues higher than $p_{\text {ground }}$ can exist with lower energy as bosons and find it energetically favorable to associate into bosons. The neutrons with momentum eigenvalues less than $p_{\text {ground }}$ would have to expend energy to associate, so they do not become bosons. The details must wait for a better understanding of the physics of high density nuclear material, so that $Q, S$, and $a$ are presently adjustable parameters in our model.

\section{Field Equations and Equations of State}

Consider a neutron star that condenses into a type II fermicon star. Groups of $S$ neutrons associate into bosons. A spinor operator, $\Psi$, represents the remaining neutrons. Curved-space Dirac matrices that obey [12],

$$
\left[\gamma^{\mu}, \gamma^{v}\right]_{+}=2 g^{\mu v} 1,
$$

define spin connection matrices and the differential operators

$$
\Gamma_{\mu} \equiv \frac{1}{4} \gamma_{v} \gamma_{; \mu}^{v} \quad \text { and } \quad D_{\mu} \equiv \partial_{\mu}+\Gamma_{\mu} .
$$

A scalar field operator, $\Phi$, represents the Bose-Einstein condensate. Let $|0\rangle$ be the boson vacuum state and $\mathrm{a}^{\dagger}$ and $\mathrm{a}$ be the ground-state creation and annihilation operators, respectively, for the bosons such that [13]

$$
\begin{gathered}
\mathrm{a}^{\dagger}\left|N_{\mathrm{b}}\right\rangle=\sqrt{N_{\mathrm{b}}+1}\left|N_{\mathrm{b}}+1\right\rangle, \\
\mathrm{a}\left|N_{\mathrm{b}}\right\rangle=\sqrt{N_{\mathrm{b}}}\left|N_{\mathrm{b}}-1\right\rangle, \\
\sqrt{N_{\mathrm{b}} !}\left|N_{\mathrm{b}}\right\rangle=\left(\mathrm{a}^{\dagger}\right)^{N_{\mathrm{b}}}|0\rangle, \\
\mathrm{N}_{\mathrm{b}}=\mathrm{a}^{\dagger} \mathrm{a} \text {, and } \\
{\left[\mathrm{a}, \mathrm{a}^{\dagger}\right]=1 .}
\end{gathered}
$$

The gravitational field depends on the Ricci tensor, $R_{\mu v}$, and the curvature scalar, $R_{\mu}^{\mu}$.

Given these relationships, the Lagrangian density operators for the neutrons, condensate, and gravitational field are

$$
\begin{gathered}
\mathcal{L}_{\mathrm{n}}=\frac{1}{2} i \hbar c g^{\mu v}\left[\bar{\Psi} \gamma_{\mu} \mathrm{D}_{v} \Psi-\left(\mathrm{D}_{\mu} \bar{\Psi}\right) \gamma_{v} \Psi\right]-m c^{2} \bar{\Psi} \Psi, \\
\mathcal{L}_{\mathrm{b}}=\hbar^{2} g^{\mu v} \partial_{\mu} \Phi^{\dagger} \partial_{v} \Phi /(2 Q s m)-\frac{1}{2} Q s m c^{2} \Phi^{\dagger} \Phi \\
-\pi m c^{2} \lambda^{2} a \Phi^{\dagger} \Phi^{\dagger} \Phi \Phi /(Q s), \text { and } \\
\mathcal{L}_{\mathrm{G}}=R_{\mu}^{\mu} \mathbf{1} /\left(2 \kappa^{2}\right),
\end{gathered}
$$

respectively, where $\lambda$ is the neutron reduced Compton length, and $\kappa^{2}$ is the relativistic gravitational constant, $\kappa^{2} \equiv 8 \pi G / c^{4}$, and $\mathbf{1}$ is the unit operator in spinor space.

The Lagrangian density operator for the particles is $\mathcal{L}_{\mathrm{P}}=\mathcal{L}_{\mathrm{n}}+\mathcal{L}_{\mathrm{b}}$ and the total Lagrangian density operator is $\mathcal{L}=\mathcal{L}_{\mathrm{n}}+\mathcal{L}_{\mathrm{b}}+\mathcal{L}_{\mathrm{G}}$.

Application of the action principle produces the necessary quantities and relationships. Given the action operators,

$$
\begin{aligned}
& \mathrm{I}=c^{-1} \int \mathcal{L} \sqrt{-g} \mathrm{~d}^{4} x \text { and } \\
& \mathrm{I}_{\mathrm{P}}=c^{-1} \int \mathcal{L}_{\mathrm{P}} \sqrt{-g} \mathrm{~d}^{4} x,
\end{aligned}
$$

the relationship,

$$
2 c \delta \mathrm{I}_{\mathrm{P}}=\int \mathrm{T}_{\mu \nu} \delta g^{\mu \nu} \sqrt{-g} \mathrm{~d}^{4} x,
$$

defines the energy-momentum tensor operator [14]. The statement,

$$
2 c \delta \mathrm{I}=\int\left(\delta \mathcal{L} \sqrt{-g} / \delta \Phi^{\dagger}\right) \delta \Phi^{\dagger} \mathrm{d}^{4} x=0,
$$

defines the field equation of motion for the condensate. The Einstein field equation follows from

$$
2 c \delta \mathrm{I}=\int\left(\delta \mathcal{L} \sqrt{-g} / \delta g^{\mu v}\right) \delta g^{\mu v} \mathrm{~d}^{4} x=0 .
$$

The current density operator of the bosons is [13]

$$
\mathrm{j}_{\mathrm{b}}^{\mu}=\frac{i}{\hbar c}\left[\frac{\partial \mathcal{L}_{\mathrm{b}}}{\partial\left(\partial_{\mu} \Phi^{\dagger}\right)} \Phi^{\dagger}-\frac{\partial \mathcal{L}_{\mathrm{b}}}{\partial\left(\partial_{\mu} \Phi\right)} \Phi\right] .
$$

From Equation (18), the condensate field equation is

$$
\lambda^{2} \Phi_{; \mu}^{; \mu}+(Q S)^{2} \Phi+4 \pi \lambda^{2} a \Phi^{\dagger} \Phi \Phi=0
$$

The line element within the star is

$$
\mathrm{d} s^{2}=g_{00}(r) c^{2} \mathrm{~d} t^{2}+g_{11}(r) \mathrm{d} r^{2}-r^{2} \mathrm{~d} \theta^{2}-r^{2} \sin ^{2} \theta \mathrm{d} \varphi^{2},
$$

Substituting Equation (22) and the separation of variables,

$$
\Phi \equiv R(r) e^{-i E t / \hbar}\left(\mathrm{a}^{\dagger}+\mathrm{a}\right) / \sqrt{8 \pi},
$$

into (21), multiplying the result on the right by $\mathrm{a}^{\dagger}+\mathrm{a}$ and then taking the mean gives

$$
\begin{aligned}
R^{\prime \prime}= & {\left[\frac{1}{2}\left(\ln g_{11}\right)-\frac{1}{2}\left(\ln g_{00}\right)-2 / r\right] R^{\prime} } \\
& +g_{11}\left\{(Q s)^{2}+\frac{3}{2} N_{\mathrm{b}} \lambda^{2} a R^{2}-E^{2} /\left[\left(m c^{2}\right)^{2} g_{00}\right]\right\} \\
& \times R / \lambda^{2}
\end{aligned}
$$

where a prime indicates a derivative with respect to $r$. Following Colpi, Shapiro, and Wasserman [15], the identities,

$$
\hat{r} \equiv r m /\left(m_{\mathrm{P}} \sqrt{\lambda a}\right) \quad \text { and } \quad \hat{R} \equiv m_{\mathrm{P}} \lambda E R \sqrt{a /\left(m^{3} c^{2}\right)},
$$

make Equation (24) dimensionless and show that a 
scattering length at least as large as the neutron reduced Compton length produces a last term at least

$\left(m_{\mathrm{P}} / m\right)^{2} \sim 10^{30}$ times larger than the first term and the left side of the equation. Neglecting these gives

$$
R=\lambda^{-1} \sqrt{2\left\{E^{2} /\left[\left(m c^{2}\right)^{2} g_{00}\right]-(Q s)^{2}\right\} /\left(3 N_{\mathrm{b}} a\right)} .
$$

Evaluating Equation (19) and applying the semiclassical approximation, we obtain

$$
R_{\mu \nu}-\frac{1}{2} R_{\xi}^{\xi} g_{\mu \nu}=\kappa^{2}\left\langle T_{\mu \nu}\right\rangle .
$$

Substituting Equation (22) into (27) gives

$$
\begin{gathered}
g_{00}^{\prime}=\kappa^{2} r g_{00} g_{11}\left\langle T_{1}^{1}\right\rangle-g_{00}\left(1+g_{11}\right) / r \\
g_{11}^{\prime}=g_{11}\left(1+g_{11}\right) / r-\kappa^{2} r g_{11}^{2}\left\langle T_{0}^{0}\right\rangle .
\end{gathered}
$$

Evaluating Equation (17) and taking the mean gives

$$
\begin{aligned}
\left\langle T_{0}^{0}\right\rangle= & i \hbar c g^{00}\left\langle\bar{\Psi} \gamma_{0} \mathrm{D}_{0} \Psi-\left(\mathrm{D}_{0} \bar{\Psi}\right) \gamma_{0} \Psi\right\rangle \\
& +\hbar^{2}\left\langle\dot{\Phi} \dot{\Phi}^{\dagger}\right\rangle /\left(2 Q s m c^{2} g_{00}\right) \\
& -\hbar^{2}\left\langle\left(\Phi^{\dagger}\right)^{\prime} \Phi^{\prime}\right\rangle /\left(2 Q s m g_{11}\right)+\frac{1}{2} Q s m c^{2}\left\langle\Phi^{\dagger} \Phi\right\rangle \\
& +\pi m c^{2} \lambda^{2} a\left\langle\Phi^{\dagger} \Phi^{\dagger} \Phi \Phi\right\rangle /(Q s) \\
\left\langle T_{1}^{1}\right\rangle= & i \hbar c g^{11}\left\langle\bar{\Psi} \gamma_{1} \mathrm{D}_{1} \Psi-\left(\mathrm{D}_{1} \bar{\Psi}\right) \gamma_{1} \Psi\right\rangle \\
& +\hbar^{2}\left\langle\Phi^{\prime}\left(\Phi^{\dagger}\right)^{\prime}\right\rangle /\left(2 Q s m g_{11}\right) \\
& -\hbar^{2}\left\langle\dot{\Phi}^{\dagger} \dot{\Phi}\right\rangle /\left(2 Q s m c^{2} g_{00}\right)+\frac{1}{2} Q s m c^{2}\left\langle\Phi^{\dagger} \Phi\right\rangle \\
& +\pi m c^{2} \lambda^{2} a\left\langle\Phi^{\dagger} \Phi^{\dagger} \Phi \Phi\right\rangle /(Q s) \\
& +\pi m c^{2} \lambda^{2} a\left\langle\Phi^{\dagger} \Phi^{\dagger} \Phi \Phi\right\rangle /(Q s) \\
\left\langle T_{2}^{2}\right\rangle= & -i \hbar c r^{2}\left\langle\bar{\Psi} \gamma_{2} \mathrm{D}_{2} \Psi-\left(\mathrm{D}_{2} \bar{\Psi}\right) \gamma_{2} \Psi\right\rangle \\
& -\hbar^{2}\left\langle\Phi^{\prime}\left(\Phi^{\dagger}\right)^{\prime}\right\rangle /\left(2 Q s m g_{11}\right) \\
+ & -i \hbar c r^{2} \sin ^{2} \theta\left\langle\bar{\Psi} \gamma_{2} \mathrm{D}_{2} \Psi-\left(\Phi^{\dagger}\right)^{\prime}\right\rangle /\left(2 Q s m g_{11}\right) \\
& \left.-\hbar^{2}\left\langle\dot{\Phi}^{\dagger} \dot{\Psi}\right\rangle /\left(2 Q s m c^{2} g_{00}\right)+\frac{1}{2} Q s m c^{2}\left\langle\Phi^{\dagger} \Psi\right\rangle\right\rangle \\
& \pi c^{2} \lambda^{2} a\left\langle\Phi^{\dagger} \Phi^{\dagger} \Phi \Phi\right\rangle /(Q s)
\end{aligned}
$$

Dots indicate derivatives with respect to time. We will now show that this energy-momentum density behaves like a perfect fluid. The density of a perfect fluid is $\rho c^{2}=\left\langle T_{0}^{0}\right\rangle$ and its pressure is
$P=-\left\langle T_{1}^{1}\right\rangle=-\left\langle T_{2}^{2}\right\rangle=-\left\langle T_{3}^{3}\right\rangle$. Ruffini and Bonazzola showed that, as long as $\rho \ll 6.56 \cdot 10^{25} \mathrm{~kg} / \mathrm{m}^{3}$, the terms with $\Psi$ behave like a perfect fluid and reduce to the form [16],

$$
\begin{gathered}
\rho=\rho_{0}(\sinh f-f) \text { and } \\
P=\frac{1}{3} \rho_{0} c^{2}\left(\sinh f-8 \sinh \frac{1}{2} f+3 f\right),
\end{gathered}
$$

where $\rho_{0}=5.72 \cdot 10^{17} \mathrm{~kg} / \mathrm{m}^{3}$. The maximum density of a stable OV star easily meets Ruffini and Bonazzola's conditions (it is $4 \cdot 10^{18} \mathrm{~kg} / \mathrm{m}^{3}$ ), so these reductions are valid.

We consider $f$, the exact form of which we obtain shortly, to be the effective field that describes a large collection of neutrons. Substituting Equations (23), (34) and (35) into (30) through (33) gives

$$
\begin{aligned}
\left\langle T_{0}^{0}\right\rangle= & {\left[N_{\mathrm{b}} m c^{2} /(32 \pi Q s)\right] } \\
& \times\left(4\left\{(Q s)^{2}+E^{2} /\left[\left(m c^{2}\right)^{2} g_{00}\right]\right\} R^{2}\right. \\
- & {\left.\left[4 / g_{11}\right]\left[\lambda R^{\prime}\right]^{2}+3 N_{\mathrm{b}} \lambda^{2} a R^{4}\right)+\rho_{0} c^{2}(\sinh f-f) . } \\
\left\langle T_{1}^{1}\right\rangle= & {\left[N_{\mathrm{b}} m c^{2} /(32 \pi Q s)\right] } \\
& \times\left(4\left\{(Q s)^{2}-E^{2} /\left[\left(m c^{2}\right)^{2} g_{00}\right]\right\} R^{2}\right. \\
& \left.+\left[4 / g_{11}\right]\left[\lambda R^{\prime}\right]^{2}+3 N_{\mathrm{b}} \lambda^{2} a R^{4}\right) \\
& -\frac{1}{3} \rho_{0} c^{2}\left(\sinh f-8 \sinh \frac{1}{2} f+3 f\right) \\
\left\langle T_{2}^{2}\right\rangle= & \left\langle T_{3}^{3}\right\rangle=-\frac{1}{3} \rho_{0} c^{2}\left(\sinh f-8 \sinh \frac{1}{2} f+3 f\right) \\
+ & {\left[N_{\mathrm{b}} m c^{2} /(32 \pi Q s)\right] } \\
\times & \left(4\left\{(Q s)^{2}-E^{2} /\left[\left(m c^{2}\right)^{2} g_{00}\right]\right\} R^{2}\right. \\
& {\left.\left[4 / g_{11}\right]\left[\lambda R^{\prime}\right]^{2}+3 N_{\mathrm{b}} \lambda^{2} a R^{4}\right) . }
\end{aligned}
$$

Substituting Equations (25) gives dimensionless forms showing that the term with $R^{\prime}$ is negligible because it is $m / m_{\mathrm{P}} \sim 10^{-15}$ times the other terms. Neglecting this term and substituting Equation (26) gives

$$
\begin{aligned}
\left\langle T_{0}^{0}\right\rangle= & {\left[4 \pi \rho_{0} c^{2} \lambda /(3 Q s a)\right] } \\
& \times\left\{(Q s)^{2}+3 E^{2} /\left[\left(m c^{2}\right)^{2} g_{00}\right]\right\} \\
& \times\left\{E^{2} /\left[\left(m c^{2}\right)^{2} g_{00}\right]-(Q s)^{2}\right\}+\rho_{0} c^{2}(\sinh f-f) .
\end{aligned}
$$




$$
\begin{aligned}
& \left\langle T_{1}^{1}\right\rangle=\left\langle T_{2}^{2}\right\rangle=\left\langle T_{3}^{3}\right\rangle \\
= & -\left[4 \pi \rho_{0} c^{2} \lambda /(3 Q s a)\right] \\
\times & \left\{E^{2} /\left[\left(m c^{2}\right)^{2} g_{00}\right]-(Q s)^{2}\right\}^{2} \\
- & \frac{1}{3} \rho_{0} c^{2}\left(\sinh f-8 \sinh \frac{1}{2} f+3 f\right) .
\end{aligned}
$$

The only two diagonal energy-momentum tensor components we need are $\left\langle T_{0}^{0}\right\rangle$ and $\left\langle T_{1}^{1}\right\rangle$ since the star's pressure is now isotropic.

Substituting Equations (39) and (41) into (28) and (29) gives

$$
\begin{aligned}
g_{00}^{\prime}= & -\frac{1}{3} \kappa^{2} r g_{00} g_{11}\left(\left\{m c^{2} /\left(8 \pi Q s \lambda^{2} a\right)\right\}\right. \\
\times & \left\{E^{2} /\left[\left(m c^{2}\right)^{2} g_{00}\right]-(Q s)^{2}\right\}^{2} \\
+ & \left.\rho_{0} c^{2}\left\{\sinh f-8 \sinh \frac{1}{2} f+3 f\right\}\right) \\
- & g_{00}\left(1+g_{11}\right) / r . \\
g_{11}^{\prime}=- & \kappa^{2} r g_{11}^{2}\left(\left\{m c^{2} /\left(24 \pi Q s \lambda^{2} a\right)\right\}\right. \\
& \times\left\{(Q s)^{2}+3 E^{2} /\left[\left(m c^{2}\right)^{2} g_{00}\right]\right\} \\
& \times\left\{E^{2} /\left[\left(m c^{2}\right)^{2} g_{00}\right]-(Q s)^{2}\right\} \\
& \left.+\rho_{0} c^{2}\{\sinh f-f\}\right)+g_{11}\left(1+g_{11}\right) / r .
\end{aligned}
$$

\section{Boundary Conditions and Condition of Chemical Equilibrium}

Beyond the radial distance where $g_{00}=E^{2} /\left(Q s m c^{2}\right)^{2}$, the condensate density becomes negligible. We will show that this is also the star's radius, $a_{\text {star }}$.

At the star's radius, the interior solutions for $g_{00}$ and $g_{11}$ must match the vacuum Schwarzschild solution. In particular, in the limit as $r$ approaches $a_{\text {star }}$ from the left,

$$
\lim _{r \rightarrow a_{\text {star }}^{-}} g_{00}=1-\kappa^{2} M c^{2} /\left(4 \pi a_{\text {star }}\right)=-1 / g_{11}\left(a_{\text {star }}\right) .
$$

Oppenheimer and Volkoff showed that the enclosed mass is related to the metric by [1]

$$
g_{11}=4 \pi r /\left[\kappa^{2} M(r) c^{2}-4 \pi r\right] .
$$

Substituting $M^{\prime}=4 \pi r^{2} \rho$ and using l'Hôpital's rule gives

$$
\lim _{r \rightarrow 0} g_{11}=-1 .
$$

The condition for chemical equilibrium mathemati- cally defines the effective neutron field; Equation (2) must hold for every point within the star.

The chemical potential of a degenerate ideal gas in flat spacetime is equal to its Fermi energy [17] and the presence of a gravitational field does not change this relationship [18]. The Fermi momentum and $f$ are related by

$$
p_{\mathrm{F}}=m c \sinh f .
$$

Combining this with the relativistic relationship, $\left(m c^{2}\right)^{2}=E_{\mathrm{F}}^{2}-\left(p_{\mathrm{F}} c\right)^{2}$, gives

$$
\mu_{\mathrm{n}}=m c^{2} \cosh f .
$$

The chemical potential of the condensate is

$$
\mu_{\mathrm{b}}=m_{\mathrm{b}} c^{2}\left(1+4 \pi \lambda_{\mathrm{b}}^{2} a\left\langle n_{\mathrm{b}}\right\rangle\right) .
$$

Substituting Equations (20) with the index $\mu=0$, (23), (26), (48), and (49) into (2) gives

$$
\begin{aligned}
f= & \cosh ^{-1}\left(Q+\left\{2 E /\left(3 s m c^{2} g_{00}\right)\right\}\right. \\
& \left.\times\left\{E^{2} /\left[\left(m c^{2}\right)^{2} g_{00}\right]-(Q s)^{2}\right\}\right) .
\end{aligned}
$$

The inverse hyperbolic cosine is not defined when its parameter is less than one. As $r$ increases, so will $g_{00}$ until $g_{00}=E^{2} /\left(Q s m c^{2}\right)^{2}$ at the star's radius. This would give

$$
f\left(a_{\text {star }}\right)=\cosh ^{-1} Q \quad \text { if } \quad Q \geq 1 .
$$

Since $Q<1$, there must be a radial distance, $a_{\text {core }}<a_{\text {star }}$, such that $f\left(a_{\text {core }}\right)=0$. As a result, type II fermicon stars share a feature with type I fermicon stars: each has a Bose-Einstein condensate halo. The difference is that type I fermicon stars have a purely neutron core while type II fermicon stars have a core that is a mixture of neutrons and a Bose-Einstein condensate. Since our model requires a mixture of the neutrons and the condensate, this is an indication of its limits. Nevertheless, we expect results which have negligible halos or halos that dominate the star to be valid.

\section{Macroscopic Quantities}

Solving Equation (45) for the enclosed mass gives

$$
M(r)=\left[4 \pi /\left(\kappa^{2} c^{2}\right)\right] r\left(1+1 / g_{11}\right) .
$$

The density and pressure are

$$
\begin{aligned}
\rho= & {\left[4 \pi \rho_{0} \lambda /(3 Q s a)\right]\left\{(Q s)^{2}+3 E^{2} /\left[\left(m c^{2}\right)^{2} g_{00}\right]\right\} } \\
& \times\left\{E^{2} /\left[\left(m c^{2}\right)^{2} g_{00}\right]-(Q s)^{2}\right\}+\rho_{0}(\sinh f-f) .
\end{aligned}
$$




$$
\begin{aligned}
P & =\left[4 \pi \rho_{0} c^{2} \lambda /(3 Q s a)\right]\left\{E^{2} /\left[\left(m c^{2}\right)^{2} g_{00}\right]-(Q s)^{2}\right\}^{2} \\
& +\frac{1}{3} \rho_{0} c^{2}\left(\sinh f-8 \sinh \frac{1}{2} f+3 f\right) .
\end{aligned}
$$

\section{Solutions}

We found numerical solutions for the differential equations of the metric components, Equations (42) and (43), by providing a value for $g_{00}$ at the center of the star and searching for a boson ground-state energy, $E$, that satisfied the limiting condition, Equation (44), for $g_{00}$ at the outer border. We abandoned solutions with masses less than $0.1 M_{\odot}$ since neutrons decay into protons, electrons, and antineutrinos in these cases. Stellar radii are measured using the Schwarzschild radial coordinate for comparison with the event horizon the star would have had if it collapsed.

In both cases, there is a maximum mass and a range of masses where two or more radii exist. Oppenheimer and Volkoff showed that the equilibrium solution with a lower density is the stable one and the one with the higher density is unstable to perturbations [1].

For example, Figure 2 compares solutions for OV stars and for type II fermicon stars whose bosons have a repulsive scattering length of one femtometer and that are formed from "strongly-bound" $(Q=0.9)$ pairs $(s=2)$ of neutrons. The critical masses are $0.710 M_{\odot}$ for $\mathrm{OV}$ and $0.428 M_{\odot}$ for fermicon stars. OV and fermicon stars with radii less than $9.11 \mathrm{~km}$ and $4.10 \mathrm{~km}$, respectively, are unstable. Note the loop at the far left of the fermicon star plot, which indicates that three equilibrium states exist between $0.241 M_{\odot}$ and $0.283 M_{\odot}$. For each mass in this range, only the state with the largest radius is stable.

The speed of sound in a medium is

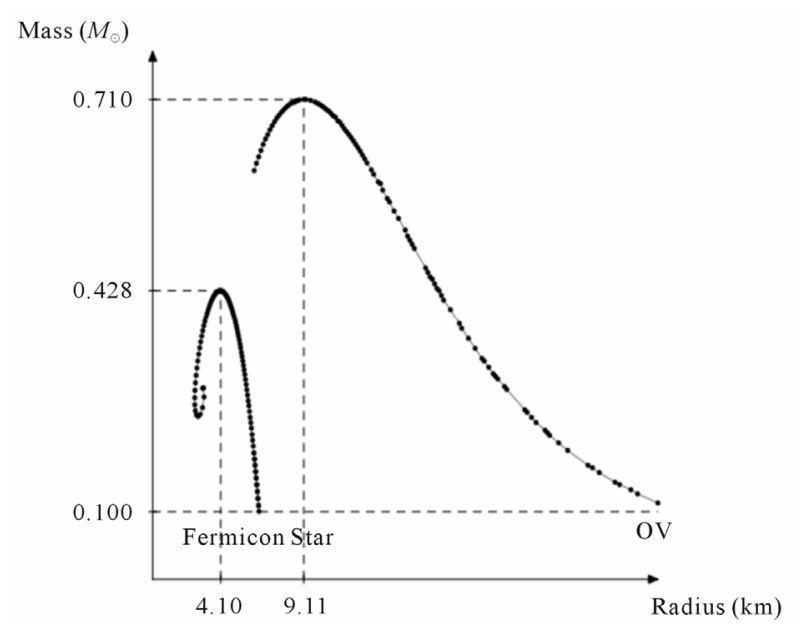

Figure 2. $Q=0.9, s=2, a=1 \mathrm{fm}$ solutions compared with OV solutions.

$$
v=\sqrt{\frac{\mathrm{d} P}{\mathrm{~d} \rho}} .
$$

We can find this speed, since Equations (53) and (54) give expressions for the density and pressure. The maximum speed of sound for a type II fermicon star with a scattering length of a femtometer is $0.55 \mathrm{c}$. The solutions obey causality.

Figure 3 shows another way to compare the two sets of solutions in Figure 2 by plotting mass-vs-core density. $\mathrm{OV}$ and fermicon stars with densities greater than 4.01 $\mathrm{kg} / \mu \mathrm{m}^{3}$ and $18.3 \mathrm{~kg} / \mu \mathrm{m}^{3}$, respectively, are unstable. To see why the fermicon stars have higher densities than OV stars, consider the asymptotic behavior of fermicon density and pressure as the scattering length gets very small or very large:

$$
\begin{aligned}
& \rho \underset{a \rightarrow 0}{[} \\
& {\left[4 \pi \rho_{0} \lambda /(3 Q s a)\right]\left\{(Q s)^{2}+3 E^{2} /\left[\left(m c^{2}\right)^{2} g_{00}\right]\right\}} \\
& \times\left\{E^{2} /\left[\left(m c^{2}\right)^{2} g_{00}\right]-(Q s)^{2}\right\} \propto 1 / a . \\
& \rho \underset{a \rightarrow \infty}{\longrightarrow} \rho_{0}(\sinh f-f) . \\
& P \underset{a \rightarrow 0}{\longrightarrow}\left[4 \pi \rho_{0} c^{2} \lambda /(3 Q s a)\right] \\
& \quad \times\left\{E^{2} /\left[\left(m c^{2}\right)^{2} g_{00}\right]-(Q s)^{2}\right\}^{2} \propto 1 / a . \\
& P \underset{a \rightarrow \infty}{\longrightarrow} \frac{1}{3} \rho_{0} c^{2}\left(\sinh f-8 \sinh \frac{1}{2} f+3 f\right) .
\end{aligned}
$$

We found that the boson ground-state energy stays close to the rest energy of a boson and that $g_{00}$ does not vary much as initial parameters are changed. All else being equal, the density and pressure of fermicon stars with small scattering lengths get larger as the scattering length gets smaller. Recall that the motivation for exploring fermicon stars was that we sought equations of

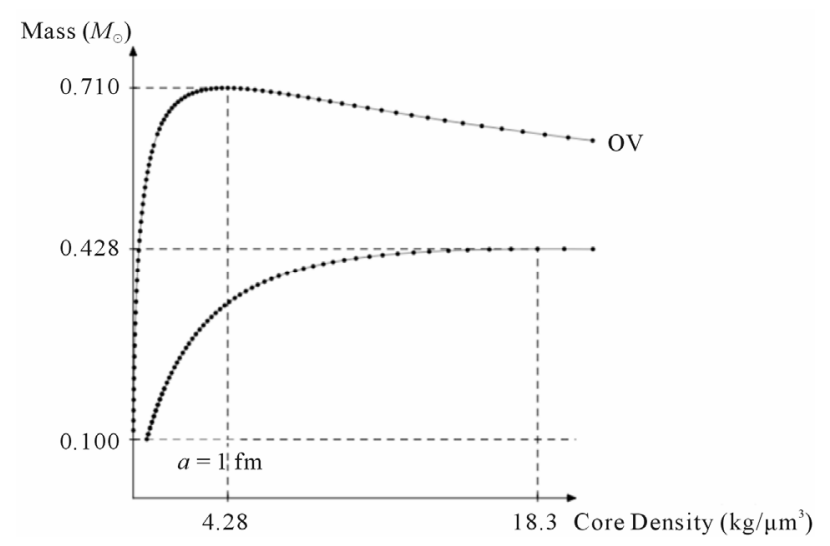

Figure 3. $Q=0.9, s=2, a=1 \mathrm{fm}$ mass-vs.-core density. 
state with lower pressure to reduce the gravitational field and increase stability. We therefore would expect lowscattering length fermicon stars to have lower critical masses than OV stars.

In contrast, fermicon stars with large scattering lengths have expressions for density and pressure identical to those of OV stars. However, $f$, which represents the presence of neutrons, is smaller in a fermicon star than in an OV star because some of the neutrons have condensed into bosons. We therefore would expect critical mass to increase as scattering length increases and sufficientlylarge scattering lengths to produce more massive stars than OV stars.

These expectations are confirmed in Figures $\mathbf{4}$ and 5, which show mass-vs-radius graphs for fermicon stars with $Q=0.9, s=2$, and scattering lengths that vary from a femtometer to 20 picometers. Figure 4 includes the plot for OV stars. Figure 5 includes the solutions for type I fermicon stars with $Q=0.9, s=2$, and $a=1$

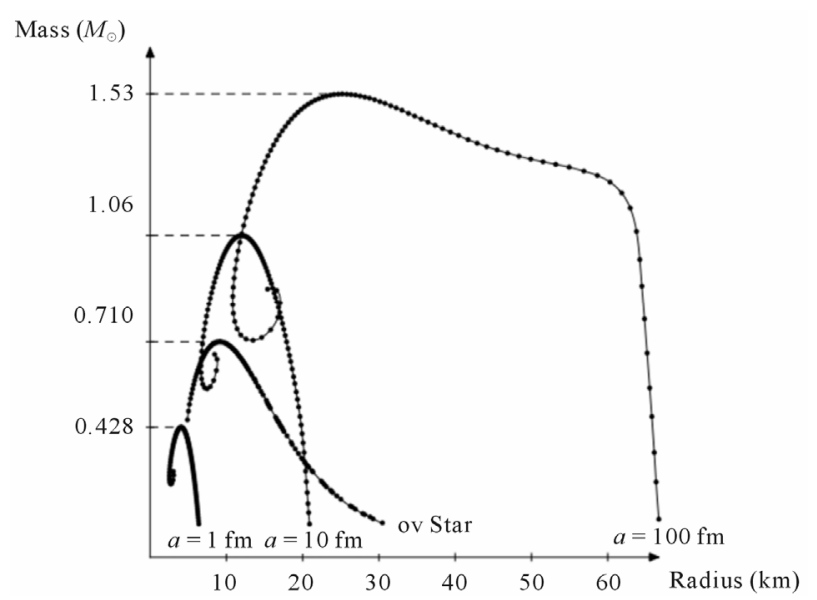

Figure 4. Type II fermicon stars with femptometer-scale scattering. All of the fermicon solutions have $Q=0.9$ and $s=$ 2 .

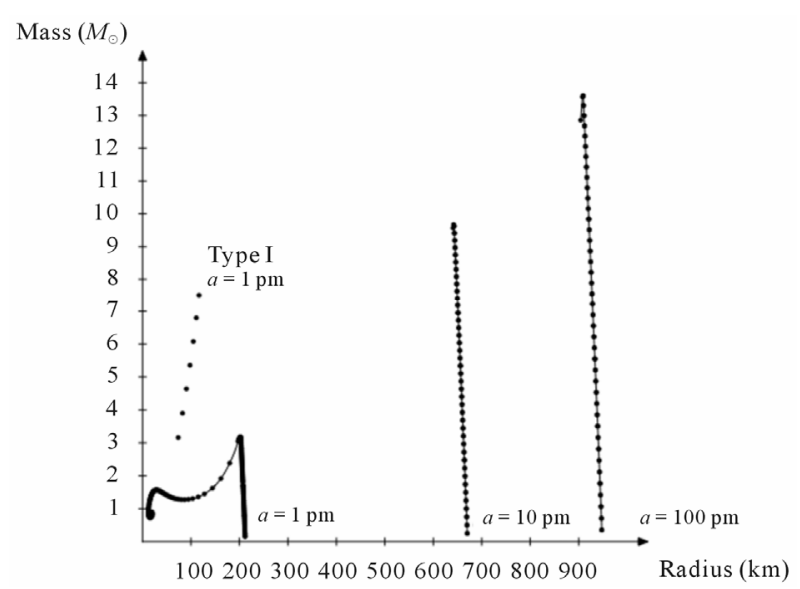

Figure 5. Type II fermicon stars with picometer-scale scattering. All solutions have $Q=0.9$ and $s=2$. pm. It only includes the stable solutions for the $a=10$ pm and $a=20 \mathrm{pm}$ stars because the time to find the unstable solutions was prohibitive.

Although the $a=1$ pm type II fermicon stars have masses higher than OV stars, they are less massive than type I stars. Type I fermicon stars have higher critical masses because they have constant density. The critical mass for a star with a constant density of $\rho$ is [19]

$$
M=4 c^{3} / \sqrt{243 \pi G^{3} \rho}=\left(3.6 M_{\odot} / \sqrt{\rho}\right) \sqrt{\mathrm{kg} / \mu \mathrm{m}^{3}} .
$$

For $\rho=4 \mathrm{~kg} / \mathrm{mm}^{3}$, this is a maximum mass of $1.8 M_{\odot}$, which is 2.6 times larger than an OV star's critical mass of $0.71 M_{\odot}$ at the same core density.

A type II fermicon star with $Q=0.9, s=2$, and $a=20 \mathrm{pm}$ has a critical mass of $13.6 M_{\odot}$. Adding neutron self-interaction and rotation to the OV star model quadruples its critical mass. If the same increase applies to type II fermicon stars, then pairing of neutrons into a Bose-Einstein condensate within neutron stars could raise their critical masses to about $50 M_{\odot}$, preventing collapse to stellar black holes. The maximum speed of sound for the stable solutions is $0.15 c$; as the solutions become less dense, the speed of sound decreases.

Returning to type II fermicon stars with one-femtometer scattering lengths, Figure 6 shows the relationship between the star's radius and its core density. Type II fermicon stars have cores consisting of a mixture of neutrons and condensate surrounded by a halo of condensate. The thickness of this halo diminishes as the core density rises. The fermicon star with a critical mass has a $4.10 \mathrm{~km}$ radius, 334 meters of which is the condensate halo. The Schwarzschild radius of a black hole with this star's mass is $1.26 \mathrm{~km}$.

Figure 7 shows the relationship between the core density and radius for type II fermicon stars with a scattering length of a picometer. These stars have larger radii consistent with their lower densities. Their radii are about 200 times larger than their Schwarzschild radii.

One feature that is difficult to see in Figure 7 is that the low-density stars are purely condensate and that the

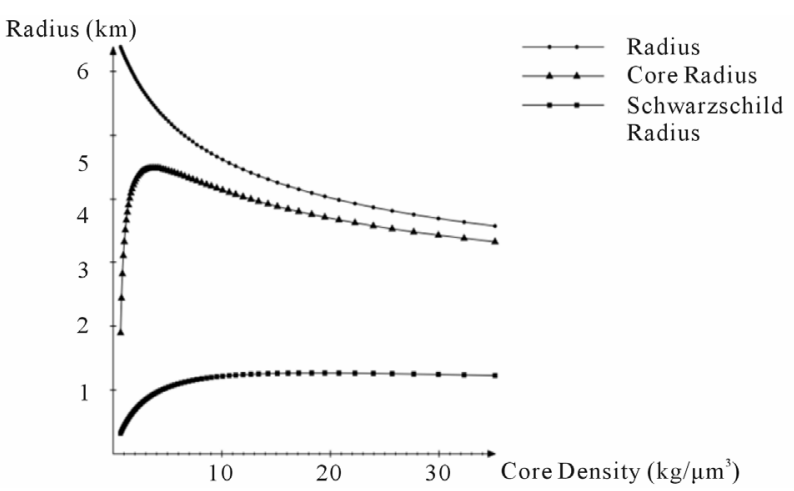

Figure 6. $Q=0.9, s=2, a=1 \mathrm{fm}$ radius-vs. -core density. 
stable stars that have neutrons are still dominated in volume by the condensate. Figure 8 shows radii for densities up to $0.00018 \mathrm{~kg} / \mathrm{\mu m}^{3}$. Below $0.000572 \mathrm{~kg} / \mu^{3}$, essentially all of the neutrons group into the condensate (more accurately, from the precision of our solutions, these stars have a core with a radius less than a millimeter). A critical-mass picometer-scaled fermicon star has a core with a radius of nine kilometers and a halo 194 kilometers thick.

Type II fermicon stars with scattering lengths comparable to their reduced Compton lengths do not have the opportunity to condense into esentially-pure condensate stars since such stars have much lower masses (the neutrons in a star below $0.1 M_{\odot}$ will decay).

If the neutrons pair into bosons with a stronger binding (e.g., $Q=0.8$ ), the boson rest energy will be smaller. As Figures 9 and 10 show, these stars are slightly more massive at lower densities than stars with weaker binding, condense completely into condensate stars at low densities, and are slightly larger than stars with weaker binding.

Neutrons may also find it energetically favorable to associate in groups of four (or higher even numbers) into bosons. Figures 11 through 12 show features of type II fermicon stars formed when four neutrons strongly group into a single boson $(Q=0.9$ and $s=4)$ and these bosons have a repulsive interaction with a scattering length of one femtometer. These stars have lower critical masses at higher densities $\left(0.153 M_{\odot}\right.$ at $\left.151 \mathrm{~kg} / \mu^{3}\right)$ than their

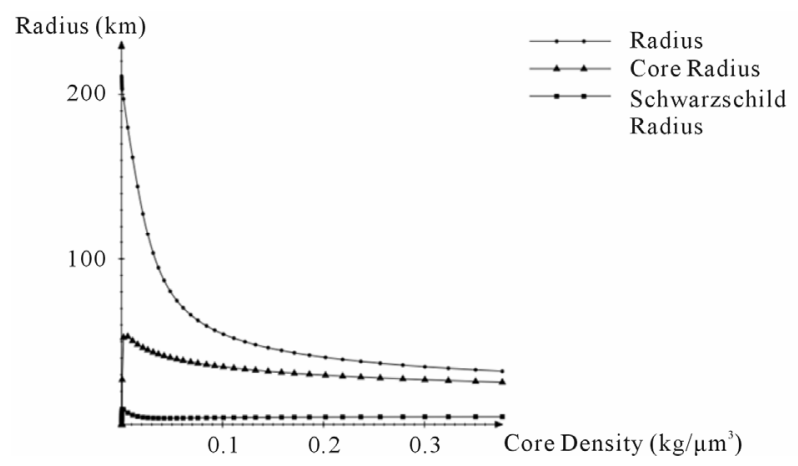

Figure 7. $Q=0.9, s=2, a=1 \mathrm{pm}$ radius-vs.-core density.

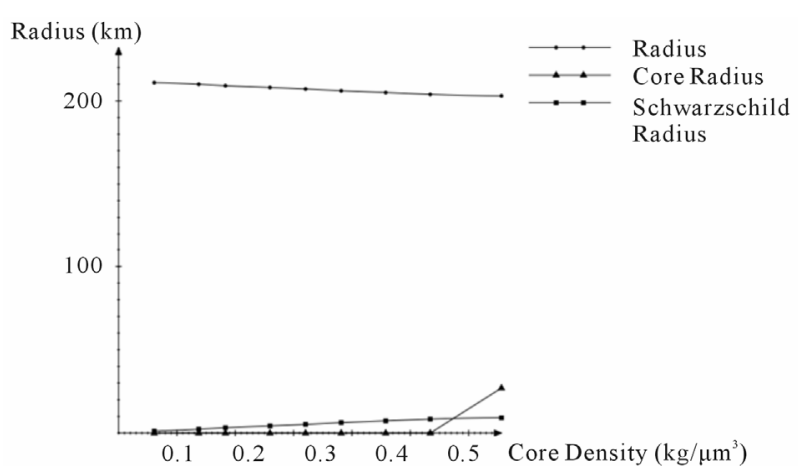

Figure 8. $Q=0.9, s=2, a=1 \mathrm{pm}$ radii at low densities.

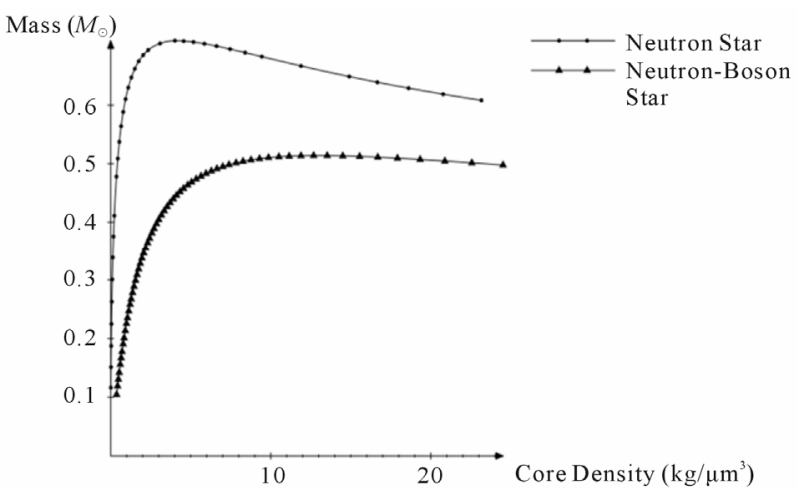

Figure 9. $Q=0.8, s=2, a=1 \mathrm{fm}$ mass-vs.-core density.

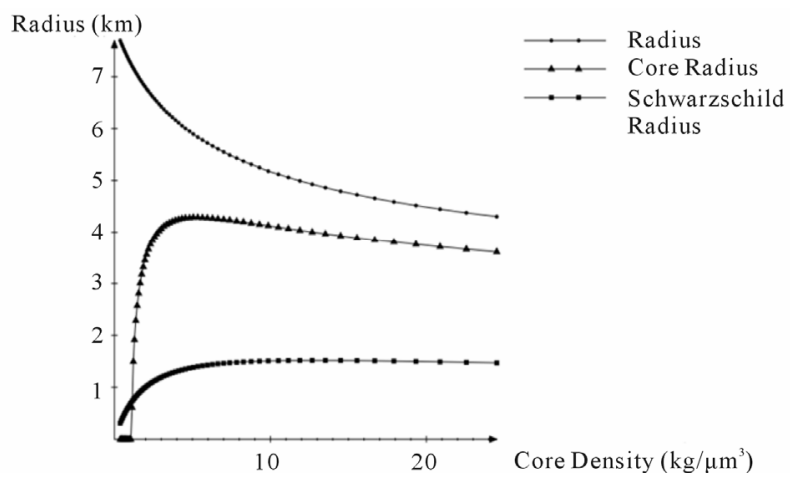

Figure 10. $Q=0.8, s=2, a=1 \mathrm{fm}$ radius-vs.-core density.

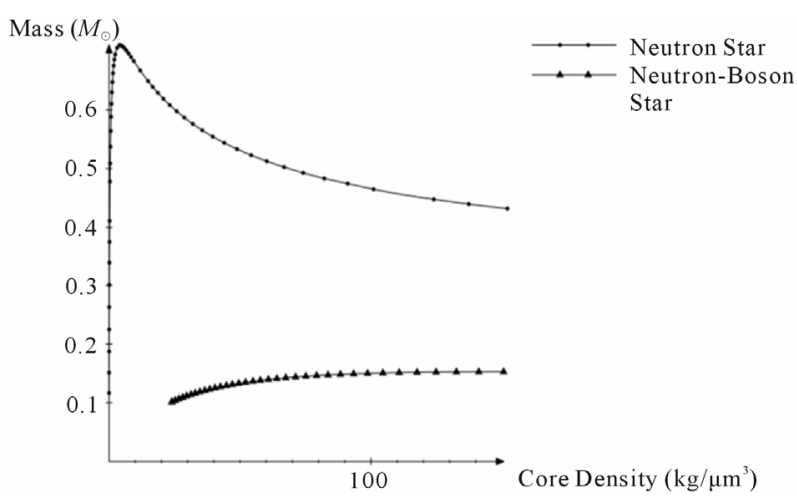

Figure 11. $Q=0.9, s=4, a=1 \mathrm{fm}$ mass-vs.-core density.

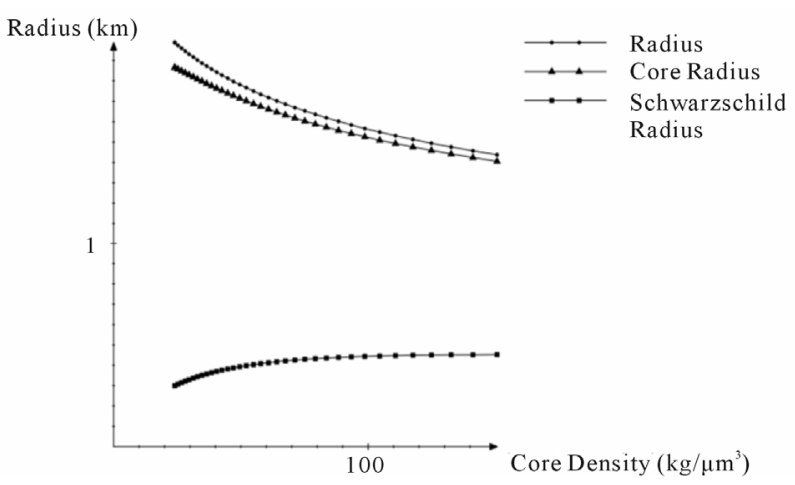

Figure 12. $Q=0.9, s=4, a=1 \mathrm{fm}$ radius-vs.-core density. 
$s=2$ counterparts $\left(0.428 M_{\odot}\right.$ at $\left.18.3 \mathrm{~kg} / \mu \mathrm{m}^{3}\right)$, and are correspondingly smaller with comparatively thinner condensate halos.

\section{Conclusion}

If strong pairwise grouping of neutrons into a BoseEinstein condensate of spin-0 bosons with scattering lengths of at least twenty picometers takes place in nonrotating massive neutron stars, these stars will become predominately condensate by volume in chemical equilibrium with their remaining neutrons. They will have a minimum radius roughly half that of the moon and have masses greater than $13 M_{\odot}$. If neutron interaction and rotation have the same effect on these stars that they have on the stars modeled by Oppenheimer and Volkoff, then their critical masses would be greater than $50 M_{\odot}$, avoiding collapse to black holes.

\section{REFERENCES}

[1] J. R. Oppenheimer and G. M. Volkoff, "On Massive Neutron Cores," Physical Review, Vol. 55, No. 4, 1939, pp. 374-381. doi:10.1103/PhysRev.55.374

[2] F. Weber, R. Negreiros, P. Rosenfield and M. Stejner, "Pulsars as Astrophysical Laboratories for Nuclear and Particle Physics," Progress in Particle and Nuclear Physics, Vol. 59, No. 1, 2007, pp. 94-113. doi:10.1016/j.ppnp.2006.12.008

[3] C. Bailyn, R. K. Jain, P. Coppi and J. A. Orosz, "The Mass Distribution of Stellar Black Holes," Astrophysical Journal, Vol. 499, 1998, pp. 367-374.

[4] W. M. Farr, N. Sravan, A. Cantell, L. Kreidberg, C. D. Bailyn, I. Mandel and V. Kalogera, "The Mass Distribution of Stellar-Mass Black Holes," Astrophysical Journal, Vol. 741, No. 103, 2011.

[5] M. P. Silverman, "Condensates in the Cosmos: Quantum Stabilization of the Collapse of Relativistic Degenerate Stars to Black Holes," Foundations of Physics, Vol. 37, 2007.

[6] P. B. Demorest, T. Pennucci, S. M. Ransom, M. S. E. Roberts and J. W. T. Hessels, "Two-Solar-Mass Neutron
Star Measured Using Shapiro Delay," Nature, Vol. 467, 2010, pp. 1081-1083.

[7] C. A. Regal, M. Greiner and D. S. Jin, "Observation of Resonance Condensation of Fermionic Atom Pairs," Physical Review Letters, Vol. 92, 2004, p. 403.

[8] J. Kinast, S. L. Hemmer, G. E. Gehm, A. Turlapov and J. E. Thomas, "Evidence for Superfluidity in a Resonantly Interacting Fermi Gas," Physical Review Letters, Vol. 92, 2004, p. 150402.

[9] R. Grimm, "Low-Temperature Physics: A Quantum Revolution," Nature, Vol. 435, No. 7045, 2005, pp. 10351036. doi: $10.1038 / 4351035 \mathrm{a}$

[10] M. W. Zwierlein, J. R. Abo-Shaeer, A. Schirotzek, C. H. Schunck and W. Ketterle, "Vortices and Superfluidity in a Strongly Interacting Fermi Gas," Nature, Vol. 435, 2005, pp. 1047-1051. doi:10.1038/nature03858

[11] L. P. Pitaevskii and S. Stringari, "The Quest for Superfluidity in Fermi Gases," Science, Vol. 298, 2002, pp. 2144-2146. doi:10.1126/science. 1080087

[12] W. Greiner, B. Müller and J. Rafelski, "Quantum Electrodynamics of Strong Fields, Texts and Monographs in Physics," Springer-Verlag, Berlin, 1985.

[13] A. Messiah, "Quantum Mechanics," Dover Publications, New York, 1999.

[14] L. D. Landau and E. M. Lifshitz, "Classical Theory of Fields," Butterworth-Heinemann, 2000, p. 292.

[15] M. Colpi, S. L. Shapiro and I. Wasserman, "Boson Stars: Gravitational Equilibria of Self-Interacting Scalar Fields," Physical Review Letters, Vol. 57, No. 20, 1986, pp. 2485-2488.

[16] R. Ruffini and S. Bonazzola, "Systems of Selfgravitating Particles in General Relativity and the Concept of an Equation of State," Physical Review, Vol. 187, No. 5, 1969, pp. 1767-1783. doi:10.1103/PhysRev.187.1767

[17] F. Schwabl, "Statistical Mechanics," Springer, Berlin, 2000.

[18] R. C. Tolman, "Relativity Thermodynamics and Cosmology," Clarendon Press, Oxford, 1934.

[19] R. Adler, M. Bazin and M. Schiffer, "Introduction to General Relativity," 2nd Edition, McGraw-Hill Book Company, New York, 1975. 